\title{
A Prospective Study on Intrapartum Risk Factors for Birth Asphyxia
}

\author{
Dr Murali Krishnan $\mathrm{P}^{1}$, Dr SreeChandu Padarthi ${ }^{2}$ \\ ${ }^{I}$ (Assistant Professor, Department Of Paediatrics, Meenakshi Medical College Hospital and Research Institute, \\ MAHER University, India) \\ ${ }_{2}^{2}$ (Post Graduate, Department Of Paediatrics, Meenakshi Medical College Hospital and Research Institute, \\ MAHER, University India)
}

\begin{abstract}
Birth asphyxia is one of the most common causes of neonatal morbidity and mortality accounting for $23 \%$ of neonatal deaths. No data is available on true incidence of birth asphyxia in tertiary care centre from Tamilnadu due to lack of prospective studies. Hence we conducted a prospective study to determine the incidence of birth asphyxia among babies exposed to intrapartum risk factors and the role of intrapartumrisk factors in birth asphyxia. Total of 674 were this study, among them 293 were exposed to any one of intrapartum risk factors and 391were not exposed to any of the intrapartum risk factors. The overall incidence of birth asphyxia was found to be 57/1000.There were total of 39(5.7\%) birth asphyxiated babies in our study. Among them 29(74.3\%) were exposed to at least one of intrapartum risk factors andother10 babies were not exposed to any of the risk factors. Hence the incidence of birth asphyxia in newborns exposed to intrapartum risk factors was $10.2 \%$ as compared to $2.5 \%$ in unexposed group. In our study we found that exposure to risk factors like induction of labor with cerviprime and oxytocin and meconium stained amniotic fluid were significantly associated with birth asphyxia.
\end{abstract}

Keywords: Birth asphyxia, Intrapartum, Newborn, Oxytocin, Seizures

\section{Introduction}

Worldwide, perinatal asphyxia is a major cause of death and of acquired brain damage in newborn infants ${ }^{[1]}$. Globally, hypoxia of the newborn ("birth asphyxia") or the fetus ("fresh stillbirth") is estimated to account for $23 \%$ of the 4 million neonatal deaths and $26 \%$ of the 3.2 million stillbirths each year ${ }^{[2,3]}$.In South East Asian region WHO has attributed $23 \%$ of neonatal deaths to birth asphyxia ${ }^{4}$. Estimates of the incidence of perinatal asphyxia vary depending on the definitions used. In resource-rich countries, the incidence of severe perinatal asphyxia (causing death or severe neurological impairment) is about $1 / 1000$ live births ${ }^{[5,6]}$. In resourcepoor countries, perinatal asphyxia is probably much more common. Data from hospital-based studies in such settings suggest an incidence of 5-10/1000 live births. However, this probably represents an underestimate of the true community incidence of perinatal asphyxia in resource-poor countries ${ }^{[7,8,9]}$. Risk factors for birth asphyxia in hospital-based settings in developing countries have been categorized into antepartum, intrapartum, and foetal causes. Hospital and home-based risk factors for birth asphyxia may be similar but such data are entirely lacking. Among the risk factors, intra partum risk factors for asphyxia are often unpredictable, for example cord prolapse and maternal haemorrhage, and can only be addressed if there is access to trained birth attendants and secondary level referral facilities which can deal with obstetric complications. This prospective study is intended to identify the intrapartum risk factors for birth asphyxia.

\section{Materials And Methods}

All newborns with documented foetal heart rate during intrapartum period and delivered during the study period between Aug 2014 and Mar 2016 were taken in to study. Fetuses exposed to intrapartum risk factors were taken as study cohort and those who are not exposed to intrapartum risk factors were taken into control cohort. We excluded babies with major congenital anomalies and extremely low birth weight babies $(<1000 \mathrm{gm})$. For both these groups, data was collected as per the proforma which included maternal and foetal/ neonatal characteristics, of intrapartum risk factors, antepartum risk factors (APH, PIH), mode of delivery and neonatal characteristics.Primary outcome as birth asphyxia and its presence or absence in study cohort and control cohort was recorded. In this study, definition of birth asphyxia is simplified for easy application and because of limited laboratory support. Moreover, strict adherence to biochemical parameters for defining birth asphyxia was not possible.Definition of birth asphyxia in our study is as following:

1. Persistence of Apgar score of $0-3$ at $>5$ min. as severe birth asphyxia and 4-6 as mild birth asphyxia.

2. Neurological manifestations in the immediate neonatal period (within 24 hours of birth) to include seizures, hypotonia, HIE and coma. 


\section{Results}

Total of 674 newborns were included in this study. Among them 293newborns were exposed to any one of the intrapartum risk factors and 381newborns were not exposed to any of the intrapartum risk factor. Out of 674 babies 39(5.7\%) fulfilled the criteria for birth asphyxia. The mean $( \pm$ SD) age of the mother in this study was $24.59( \pm 3.6)$ years. Antenatal factors like APH, PIH, diabetes mellitus and anaemia were present in 9(13\%), 119(17.7\%), 25(3.7) and 105(15.6\%) babies respectively.

In this study normal vaginal delivery and emergency LSCS accounted for 293(43.45) and 344(51.03\%) of the total deliveries. Assisted breech, forceps, elective LSCS and vacuum delivery were conducted in $26(3.85 \%), 9(0.01 \%), 3(0.004 \%)$ and $2(0.003 \%)$ pregnancies respectively. Out of 674 babies born, $524(77.7 \%)$ of babies were term and 150(22.3) were preterm. 349(51.78\%) babies were male and 325(48.22) babies were female in this study. Male to female ratio in this study was found to be 1.07:1. Low birth weight babies accounted for $21.51 \%$ of babies born. There were 24 twin pregnancies in our study.

Table No.1 shows distribution of maternal and foetal characteristics among study cohort and control cohort. Mean $( \pm \mathrm{SD})$ maternal age and mean $( \pm \mathrm{SD})$ birth weight of babies in study cohort and control cohort found to be $24.5( \pm 3.6)$ years and $2692.91 \mathrm{gm}( \pm 534.42), 24.5( \pm 3.5)$ years and $2719.34 \mathrm{gm}( \pm 541.81)$ respectively. Their respective $\mathrm{p}$ values were found to be 1.0 and 1.85 and statistically not significant. No significant difference was found in the distribution of factors between study cohort and the control cohort except multiple births which may act as a confounding factor for birth asphyxia in these 2 groups.

Table No.1 Comparison of maternal characteristics, antenatal risk factors and foetal risk factors between control cohort and study cohort

\begin{tabular}{|c|c|c|c|c|c|c|}
\hline S.NO & Characteristics & $\begin{array}{l}\text { NO. of babies exposed to } \\
\text { intrapartum risk factors } \\
(\mathrm{n}=293)\end{array}$ & $\%$ & $\begin{array}{l}\text { No. of babies not } \\
\text { exposed to intrapartum } \\
\text { risk factors }(n=381)\end{array}$ & $\%$ & $\begin{array}{c}P \\
\text { Value }\end{array}$ \\
\hline 1 & $\begin{array}{l}\text { Parity } \\
\text { Primi } \\
\text { Multi }\end{array}$ & $\begin{array}{l}154 \\
139\end{array}$ & $\begin{array}{l}52.5 \\
47.5\end{array}$ & $\begin{array}{l}178 \\
203\end{array}$ & $\begin{array}{l}46.7 \\
53.3\end{array}$ & 0.13 \\
\hline 2 & APH & 6 & 2.0 & 3 & 0.7 & 0.15 \\
\hline 3 & PIH & 58 & 19.8 & 61 & 16.0 & 0.20 \\
\hline 4 & $\begin{array}{l}\text { Gestational Age } \\
\text { Term } \\
\text { Preterm }\end{array}$ & $\begin{array}{c}237 \\
56\end{array}$ & $\begin{array}{l}80.9 \\
19.1\end{array}$ & $\begin{array}{c}287 \\
94\end{array}$ & $\begin{array}{r}75.3 \\
24.7\end{array}$ & 0.08 \\
\hline 5 & LBW & 67 & 22.9 & 78 & 20.4 & 0.45 \\
\hline 6 & Multiple births & 30 & 10.2 & 18 & 4.7 & 0.005 \\
\hline 7 & $\begin{array}{l}\text { Sex } \\
\text { Male } \\
\text { Female }\end{array}$ & $\begin{array}{l}156 \\
137\end{array}$ & $\begin{array}{l}53.2 \\
46.8\end{array}$ & $\begin{array}{l}193 \\
188\end{array}$ & $\begin{array}{l}50.7 \\
49.3\end{array}$ & 0.5 \\
\hline
\end{tabular}

Table No.2shows frequency of intrapartum risk factors in study population. For induction and acceleration oxytocin and / or cerviprime was used. Acceleration was the commonest indication to start on oxytocin infusion and/ or cerviprime insertion in 128(19\%) mothers. Induction with cerviprime alone was done in $40(5.8 \%)$ mothers in this study. In another 20(2.9\%) mothers induction was done only with oxytocin. Out of $188(27.9 \%)$ deliveries in which oxytocin and / or cerviprime was used, 126(67\%) had successful vaginal delivery and 62(33\%) were delivered by emergency LSCS for varying indications including failure of induction and failure of secondary maternal forces.

Meconium stained amniotic fluid was present in 68(10.08\%) deliveries out of which 28(41.1\%) babies were found to be non- vigorous and $40(58.9 \%)$ were vigorous babies.In this study cephalic presentation was present in $91.5 \%$ and $8.5 \%$ presented with breech. No other abnormal presentation was found. In 239(35.3\%) membrane was not ruptured at all. In rest of the mothers artificial rupture of membrane was done in $128(19.0 \%)$ and spontaneous rupture was present in 308(45.7\%). PROM membrane was found in 64(20.7\%) cases. 160 $(23.7 \%)$ mothers did not develop labor pain at all. All of them delivered by emergency LSCS for varying indications.

Table No.2also shows the RR, 95\% CI, and p value for intrapartum risk factors. Among intrapartum risk factors induction of labor with oxytocin and cerviprime, and meconium stained amniotic fluid were found to have statistically significant role in birth asphyxia with RR (95\% CI) of 3.35(1.41-1.82), 4.09(1.99-7.91) and $10.48(5.99-18.04)$ respectively. $\mathrm{P}$ value for these factors were found to be $<0.05$, which is statistically significant. Hence babies born out of labor induced by oxytocin orcerviprime and with MSAF were 3.35,4.1 and 
10.5 times higher risk of suffering from birth asphyxia respectively. Antepartum hemorrhage is the only factor found to be statistically significant among antenatal risk factors with RR of 8.41(95\% CI, 3.78-18.68) PIH, GDM and anemia were not found to be significantly associated with birth asphyxia. Both low birth weight and multiple births were not significantly associated with birth asphyxia in this study. None of the twin babies suffered birth asphyxia in this study.

Out of 674 deliveries, 39(5.7\%) babies suffered birth asphyxia. Among babies who were exposed to intrapartum risk factors, $29(10.2 \%)$ babies suffered birth asphyxia.Among the control group only $10(2.5 \%)$ babies suffered birth asphyxia. Babies exposed to intrapartum risk factors had 3.8 times higher risk of developing birth asphyxia than those babies who were not exposed.

Table 2: Risk factor analysis- Intrapartum risk factors

\begin{tabular}{|c|c|c|c|c|c|c|c|}
\hline S. No & Risk factor & No. of babies & Percentage & $\overline{\mathbf{R R}}$ & $95 \% \mathrm{CI}$ & P value & Remarks \\
\hline 1 & Abnormal presentation & 57 & $8.5 \%$ & 1.24 & $0.46-3.35$ & 0.679 & Not significant \\
\hline 2 & Cord prolapse & 7 & $1.0 \%$ & 2.50 & $0.40-15.78$ & 0.333 & Not significant \\
\hline 3 & PROM & 64 & $9.5 \%$ & 1.72 & $0.75-3.95$ & 0.201 & Not significant \\
\hline 4 & $\begin{array}{l}\text { Induction of labor with } \\
\text { Oxytocin }\end{array}$ & 28 & $2.9 \%$ & 3.35 & $1.41-1.82$ & 0.01 & Significant \\
\hline 5 & $\begin{array}{l}\text { Induction of labor with } \\
\text { Cerviprime }\end{array}$ & 40 & $5.8 \%$ & 4.1 & $1.99-7.91$ & 0.000 & Significant \\
\hline 7 & $\begin{array}{l}\text { Meconium } \\
\text { amniotic fluid }\end{array}$ & 68 & $10.1 \%$ & 10.5 & $5.99-18.04$ & 0.000 & Significant \\
\hline
\end{tabular}

\section{Discussion}

The Overall incidence of birth asphyxia in this study was found to be 57/1000 live births. Though it is higher when compared to other studies where they reported the prevalence of birth asphyxia as 26.9/1000 live births. The higher incidence in our study could be explained by large number of deliveries conducted and referral of large number of complicated pregnancies to this center unlike other hospitals from where previous studies were published ${ }^{[10]}$.Comparison of basic demographic profile, antenatal risk factors and foetal risk factors between study group and control group showed that they are comparable except multiple births. But none of the twin babies suffered birth asphyxia in this study. This could be explained by close monitoring and early intervention in case of multiple births.

We found significantly higher incidence of birth asphyxia among newborns exposed to any one of the intrapartum risk factors when compared to newborns not exposed to any of the intrapartum risk factors. This is consistent with studies done by S. Dongal, et al from Nepal and Nilufar, Shireen, et al from Bangladesh ${ }^{[10,11]}$.

Meconium staining of amniotic fluid was present in 68(10.8\%) deliveries out of which28( 41.1\%) were non vigorous babies, which correlates with study done by Vineet Gupta, et al at institute of medical sciences, Banaras Hindu university, Varanasi ${ }^{[12]}$.

Among intrapartum risk factors induction of labor with cerviprime, oxytocin and MSAF were found to be independently associated with increased risk of birth asphyxia in newborn babies . Similar findings were observed in studies done by Kaye, et al from Uganda and NiluferShireen, et al $^{[12,13]}$. Though association of birth asphyxia with malpresentation, cord prolapse, PROM was not statistically proven in this study their role in birth asphyxia cannot be ignored without large scale prospective studies. Almost all the mothers with these risk factors were delivered by emergency LSCS. This could probably explain the lower incidence of birth asphyxia in these babies.

Antepartum hemorrhage was found to be significantly associated with birth asphyxia. Babies exposed to APH had 8 times higher risk suffering from birth asphyxia, finding which is comparable to other large scale studies ${ }^{[11]}$ pregnancy induced hypertension was not found to have statistically significant association with birth asphyxia in this study unlike other studies ${ }^{[11,14]}$.

Though $22.3 \%$ babies in this study were preterm we could not find any statistically significant association between low birth weight and birth asphyxia. There were 24 twin pregnancies in this study. Almost all of them were delivered by emergency LSCS and no birth asphyxia was found in all 48 babies unlike other studies ${ }^{[12,15]}$. This could be explained by close monitoring of twin pregnancies and early decision to go for emergency LSCS before any complications occur. 
Unlike previous studies our study was done in prospective manner, so relative risk of having birth asphyxia among babies exposed to intrapartum risk factors was obtained. This can be considered as preliminary study for analysis of risk factors which were not found to be significantly associated with birth asphyxia with large scale prospective studies in future. Studies based on individual risk factor may throw more light on this aspect.

Recognition and close monitoring of at risk pregnancies will significantly reduce the incidence of birth asphyxia and birth asphyxia related morbidity and mortality.

\section{Conclusion}

In our study we concluded that intrapartum risk factors are significantly associated with birth asphyxia. Based on our study induction of labor with cerviprime, oxytocin and meconium stained amniotic fluid is associated with increased risk of birth asphyxia. Anticipation of risk factors and timely intervention will significantly reduce the incidence of birth asphyxia and its related mortality and morbidity.

\section{References}

[1]. Jones G, Steketee RW, Black RE, et al. How many child deaths can we prevent this year? Lancet 2003;362:65-71.

[2]. Lawn J, Shibuya K, Stein C. No cry at birth: Global estimates of intrapartum stillbirths and intrapartum-related neonatal deaths. Bull World Health Organ 2005;83:409-417.

[3]. Stanton C, Lawn JE, Rahman H, Wilczynska-Ketende K, Hill K. Stillbirth rates: Delivering estimates in 190 countries. Lancet 2006;367: 1487-1494.

[4]. Lawn JE, Cousens SN, Wilczynska K. Estimating the causes of four million neonatal deaths in the year 2000: Statistical annexthe World Health Organization; 2005.

[5]. Levene ML, Kornberg J, Williams TH. The incidence and severity of post-asphyxial encephalopathy in full-term infants. Early Hum Dev1985;11:21-26.

[6]. Thornberg E, Thiringer K, Odeback A, et al. Birth asphyxia: incidence, clinical course and outcome in a Swedish population. ActaPaediatr 1995;84:927-932.

[7]. Airede AI. Birth asphyxia and hypoxic-ischaemic encephalopathy: incidence and severity. Ann Trop Paediatr 1991;11:331-335.

[8]. Oswyn G, Vince JD, Friesen H. Perinatal asphyxia at Port Moresby General Hospital: a study of incidence, risk factors and outcome. P N G Med J 2000;43:110-120.

[9]. Jones G, Steketee RW, Black RE, et al. How many child deaths can we prevent this year? Lancet 2003;362:65-71.

[10]. S. Dongal, J Singh, S Shrestha, A Shakya. Clinicl profile of birth asphyxia in Dhulikal hospital: Retrospective study. J NepPaedtr sol2010; 30(3): 141-146.

[11]. NiluferShireen ,NazamunNahar, AH Mollah.risk factor and short term outcome of birth asphyxiated babies in Dhaka medical college Hospital. Bangladesh J Child health 2009; 33(3): 83-89.

[12]. Vinetgupta, B. D Bhatia and O.P. Mishra. Meconium stained amniotic fluid:Antenatal,intrapartum and neoanatal attributes: Indian Paediatrics. 1996; 33(4): 293-297.

[13]. Kaye, Antenatal and intrapartum risk factors for birth asphyxia anomg obstetric referrals in Mulango Hospital, Kampala, Uaganda. East African Medical Journal 2003; 80(3): 140-143

[14]. BahubaliGane, Vishnu Bhat B, RamachandranRao, Nandakumar S, Adhisivam B, Rojo Joy, Prasad P, Shruthi S. Antenatal and intrapartum risk factors for perinatal asphyxia: A case control study. Curr. Pediatr Res 2013; 17(2) 119-122.

[15]. Aslam et al. Risk factors for birth asphyxia.Italian journal of Paediatrics 2014; 1-9 